\title{
Absolute X-Ray Yields from Laser-Irradiated, Ge-doped Aerogel Targets
}

K. B. Fournier, M. Tobin, J. F. Poco, K. Bradley, C. A. Coverdale, D. Beutler, T. Vidnovic III, S. B. Hansen, M. Severson, E. A. Smith, D. L. Reeder

July 7,2005

LASER-GENERATED, SYNCHROTRON, AND OTHER LABORATORY X-RAY AND EUV SOURCES, OPTICS, AND APPLICATIONS

San Diego, CA, United States

July 31, 2005 through August 4, 2005 
This document was prepared as an account of work sponsored by an agency of the United States Government. Neither the United States Government nor the University of California nor any of their employees, makes any warranty, express or implied, or assumes any legal liability or responsibility for the accuracy, completeness, or usefulness of any information, apparatus, product, or process disclosed, or represents that its use would not infringe privately owned rights. Reference herein to any specific commercial product, process, or service by trade name, trademark, manufacturer, or otherwise, does not necessarily constitute or imply its endorsement, recommendation, or favoring by the United States Government or the University of California. The views and opinions of authors expressed herein do not necessarily state or reflect those of the United States Government or the University of California, and shall not be used for advertising or product endorsement purposes. 


\title{
Absolute x-ray yields from laser-irradiated, Ge-doped aerogel targets
}

\author{
K.B. Fournier ${ }^{a}$, M. Tobin ${ }^{a}$, J.F. Poco ${ }^{a}$, K. Bradley ${ }^{a}$, C.A. Coverdale $^{b}$, D.E. Beutler ${ }^{b}$, \\ T. Vidnovic, III ${ }^{a}$, S.B. Hansen ${ }^{a}$, M. Severson ${ }^{b}$, E.A. Smith ${ }^{c}$, D.L. Reeder ${ }^{c}$ \\ ${ }^{a}$ Lawrence Livermore National Laboratory, Livermore, CA, USA; \\ ${ }^{b}$ Sandia National Laboratory, Albuquerque, NM, USA; \\ ${ }^{c}$ KTech Corporation, 1300 Eubank Blvd. S.E., Albuquerque, NM, USA
}

\begin{abstract}
We have measured the production of $h \nu \geq 10 \mathrm{keV}$ x rays from low-density, Ge-doped aerogel targets at the OMEGA laser (Laboratory for Laser Energetics, University of Rochester). The targets were $1.2 \mathrm{~mm}$ long by $1.5 \mathrm{~mm}$ diameter beryllium cylinders filled with Ge-doped (20 atomic percent) $\mathrm{SiO}_{2}$ aerogel. The doped-aerogel density was 4.8 or $6.5 \mathrm{mg} / \mathrm{cc}$. These targets are a major advance over previous doped aerogels: instead of suspending the dopant in the $\mathrm{SiO}_{2}$ matrix, the Ge atoms, with chemistry similar to $\mathrm{Si}$, are incorporated directly in the matrix. Forty beams of the OMEGA laser $(\lambda=351 \mathrm{~nm})$ illuminated the two cylindrical faces of the target with a total power of approximately $18 \mathrm{TW}$. The laser interaction strongly ionizes the target $\left(n_{\epsilon} / n_{c r}\right.$ $\leq 0.1-0.2$, and allows the laser-bleaching wave to ionize supersonically the high- $Z$ emitter ions in the sample. Ge K-shell x-ray emission was spectrally resolved with a two-channel crystal spectrometer and recorded with temporal resolution with a set of calibrated photoconductive devices (PCDs). The heating of the target was imaged with a gated ( $60 \mathrm{ps}$ time resolution) x-ray framing camera, filtered to observe $>4 \mathrm{keV}$. 2-D radiativehydrodynamic calculations predict rapid and uniform heating over the whole target volume with minimal energy losses into hydrodynamic motion. The calculations predict $150-200 \mathrm{~J}$ of x-ray energy output with $h \nu \geq 10 \mathrm{keV}$. Good agreement between measurements and the calculations is found.
\end{abstract}

Keywords: X-ray source, Ge K-shell x rays, Low-density aerogel, Lasers

\section{INTRODUCTION}

Bright, multi-keV x-ray sources are essential for radiography of inertial-confinement fusion (ICF) experiments. In this context, there are two parameters of importance: the energy of the emitted photons, which determines how penetrating the $\mathrm{x}$ rays are for radiographic purposes, and the brightness of the source, which determines the ability of a radiograph to detect small features. As ICF experiments continue to get larger, such as those that will be carried out at the National Ignition Facility at Lawrence Livermore National Laboratory or at the French Commissariat à l'Energie Atomique's (CEA) Laser MegaJoule, and compression in fuel capsules gets higher, sources for $\mathrm{x}$-ray backlighting will have to have harder-photon spectra. ${ }^{1}$ For a given laser energy available to drive the multi-keV x-ray-backlight source, the only way for the source to get brighter is for it to convert the laser to $\mathrm{x}$ rays more efficiently. However, as the photon energy of the $\mathrm{x}$-ray spectrum increases, the efficiency of the x-ray emitter drops sharply for solid targets. ${ }^{2}$ Underdense sources, where the electron density is less than $20 \%$ of the laser's critical density $\left(n_{e} / n_{c r}<0.2\right)$, are inherently more efficient than traditional solid-foil backlighter targets because the underdense nature of the target allows the laser to burn through the target supersonically. ${ }^{3}$ The laser's energy thus goes to ionizing and heating x-ray emitting ions rather than ablatively removing material from a bulk target; the laser interacts with the underdense x-ray emitting plasma locally, rather than depositing its energy in the blow-off material far from the bulk of a massive, solid target.

We have been developing and characterizing laser-driven, underdense $\mathrm{x}$-ray sources that are highly efficient in recent years. ${ }^{4-7}$ CEA researchers have also recently had great success efficiently making multi-keV x rays from

Further author information: (Send correspondence to K.B.F.)

K.B.F.: E-mail: fournier2@llnl.gov, Telephone: 19254236129 
pre-pulsed foils. ${ }^{8,9}$ Part of the present research has been to find materials that are solid at room temperature, can be shaped and handled easily, and are efficient producers of high-energy x rays. Typically, gas targets have been used to produce high x-ray fluences at laser facilities. ${ }^{4,7}$ The experiments described here used low-density, germanium-doped aerogel as the converter material. The aerogel is solid at room temperature and, if stored carefully, appears to have an indefinite shelf life. The target is a significant advance in both the chemistry of aerogel materials and in the fabrication of laser-driven x-ray sources. In addition to the radiography applications mentioned above, plasma-radiation sources can be used for x-ray radiation effects testing. Exo-atmosphereic radiation threats create stringent operational hardness requirements, which must be validated with tests on adequate simulators. The targets of the present work obtained about $0.9 \%$ conversion efficiency, much better than solid targets that generate $\mathrm{x}$ rays in this energy range, in a recent series of $\mathrm{x}$-ray effects tests. A number of different objects were placed close to the doped aerogel and the objects responses to the generated $x$ rays were measured. The $\mathrm{x}$ rays produced thermo-mechanical shocks in aluminum plates and induced electrical currents in some of the test objects.

The following sections present our experimental set-up, the targets that produced the $x$-ray sources, and analysis of our data. Simulations of the x-ray output from the target are also presented; we find that our lowdensity aerogel targets convert $\approx 0.9 \%$ of the laser's energy into $\mathrm{x}$ rays between 4.5 and $15 \mathrm{keV}$, with an average spectral energy of $7.1 \mathrm{keV}$.

\section{EXPERIMENTAL SET-UP}

In this section, we describe the laser facility at which the current series of experiments was performed, the x-ray source, and the diagnostics of the x-ray output. The OMEGA target chamber is nearly 3.2 meters in diameter. During campaigns, it is kept at high vacuum $\left(\approx 2-5 \times 10^{-5}\right.$ Torr $)$. There are six ten-inch manipulators (TIMs) attached to the chamber, each of which is separated from the vacuum by a flapper valve. A boat on rails can be extended from the TIM into the chamber. All diagnostic instruments were designed to fit in these boats, which allowed changes to diagnostics between shots without having to break the vacuum of the main target chamber. The time to pump one of the TIMs is approximately 5 minutes. High-precision mechanical pointers were made for each diagnostic to place them at the desired standoff distance from the x-ray source and to be sure there was no interference with the laser beams.

\subsection{The Laser}

The OMEGA laser (Laboratory for Laser Energetics, University of Rochester) is a 60 beam Nd:glass laser that can deliver up to $30 \mathrm{~kJ}$ of $3 \omega(\lambda=351 \mathrm{~nm})$ light to the target chamber center. ${ }^{10}$ Pulse shapes can be tailored to deliver the energy in flattop pulses or pulses with rising or falling ramps. Typically, pulse lengths range from one to three nanoseconds. Individual beams can be delayed relative to others by arbitrary amounts up to 6 ns. The best focus available for each beam is a spot approximately $50 \mu \mathrm{m}$ in diameter, but can be made larger as required. Several techniques exist for smoothing the intensity distribution across the laser spot, including inserting phase plates in the focusing optics, adding spectral noise to the seed pulse, and separating, then overlapping, the different polarization components in the beam (distributed polarization rotators or DPRs). It is this latter method that we used in the present experiments. The use of the DPRs means that the individual beam footprints are nearly $80 \mu \mathrm{m}$ diameter at best focus.

For the arrangement of the target in this series of shots, we were able to irradiate our x-ray source with 40 beams ( 20 per cylindrical face of the target) that strike the target in three cones of beams at $21.42,42.00,58.85^{\circ}$, respectively (Fig. 1). The most-shallow-angle cone of beams (cone 1) was focused to a point $700 \mu \mathrm{m}$ in front of the target's face and diverged onto the target material. The two steeper cones of beams ( 2 and 3 ) were focused at points inside the target; the footprints of the beams on the cylindrical faces of the target in cones 1,2 and 3 had $\approx 314,221$ and $334 \mu \mathrm{m}$ diameters, respectively. The resulting vacuum laser intensity where all 20 beams overlap on a single face of the target is $\approx 1.4 \times 10^{16} \mathrm{~W} / \mathrm{cm}^{2}$ in an $\approx 500 \mu \mathrm{m}$ spot. All laser pulses were $\approx 1$ ns square pulses with $150 \mathrm{ps}$ rising and falling edges (pulse shape SG1018). For four of the six shots conducted, all 40 beams were incident at $\mathrm{t}=0$, while for two of the shots $(39157,39158)$, the 10 cone 2 beams and 20 cone 3 beams were delayed by 250 and $500 \mathrm{ps}$, respectively, with respect to the cone 1 beams. The total energy delivered to the target and vacuum laser intensity on each face of the target for each shot in this series are listed in Table 1. 


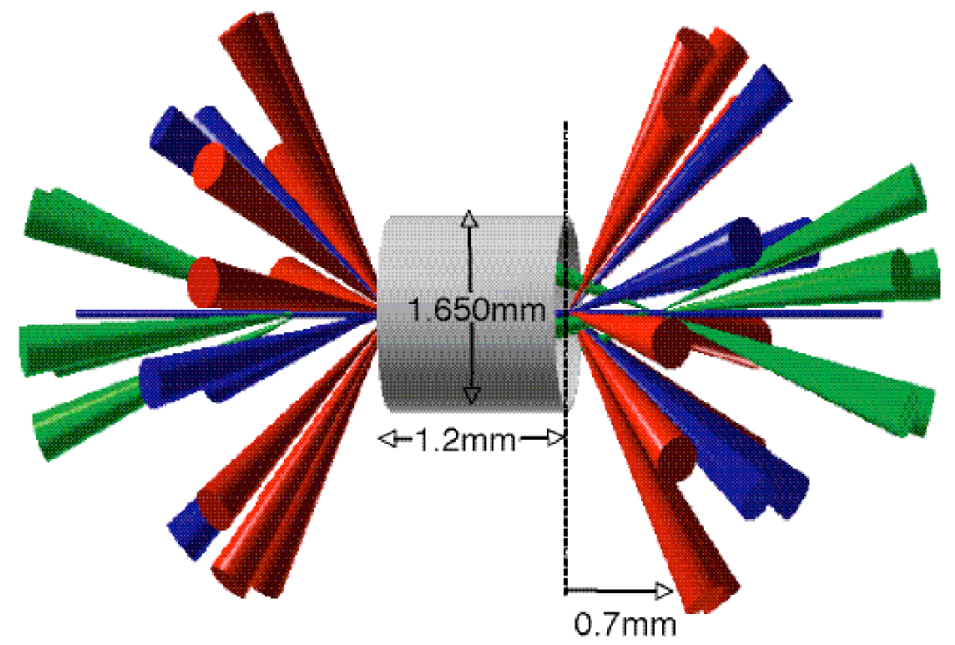

Figure 1. The $75 \mu \mathrm{m}$ thick Be cylinder that holds the underdense aerogel targets used in the current experiments. Also shown are the three cones of beams at the OMEGA laser that irradiate the two faces of the target.

Table 1. Shot number and target density for each shot in the x-ray source-development series described here. Also listed are the total energy delivered to the target, the vacuum laser intensity across an $\approx 500 \mu \mathrm{m}$ diameter spot on the target's face, and the measured energy reflected back into the optics for beams 25 and 30 in both the SRS and SBS channels, listed as a percentage of the energy in the individual beams.

\begin{tabular}{lccccccc}
\hline \hline shot no. & $\rho$ & Energy & Intensity & \multicolumn{2}{c}{ \% SRS } & \multicolumn{2}{c}{ \% SBS } \\
& $(\mathrm{mg} / \mathrm{cc})$ & $(\mathrm{kJ})$ & $\left(10^{16} \mathrm{~W} / \mathrm{cm}^{2}\right)$ & B25 & B30 & B25 & B30 \\
\hline 39152 & 4.8 & 17.294 & 1.33 & 0.86 & 1.13 & 2.84 & 6.29 \\
39154 & 4.8 & 18.374 & 1.41 & 0.51 & 2.93 & 5.11 & 0.00 \\
39156 & 4.8 & 18.211 & 1.40 & 1.60 & 2.17 & 1.39 & 3.76 \\
39157 & 4.8 & 18.816 & 1.45 & 1.04 & 0.76 & 2.70 & 5.59 \\
39153 & 6.5 & 17.461 & 1.33 & 0.58 & 0.70 & 2.82 & 3.82 \\
39158 & 6.5 & 18.613 & 1.43 & 0.57 & 0.69 & 4.24 & 4.87 \\
\hline \hline
\end{tabular}

Because of the fast-rising edge of the laser pulse, it was predicted that all of diagnostic data would have to be acquired with high sampling rates and large bandwidth to preserve high-frequency components. To this end, six TDS6804 (Tektronics) oscilloscopes were rented, each of which has four channels that sample at $20 \mathrm{Gsamples} / \mathrm{s}$ with $8 \mathrm{GHz}$ bandwidth. This proved to be adequate.

Energy can be reflected from the target by laser-driven instabilities such as stimulated Raman scattering (SRS), in which the incident electromagnetic wave of the laser resonantly decays into a plasma electron wave and a reflected wave, and by stimulated Brillouin scattering (SBS), in which the laser wave decays into ion acoustic waves in the plasma and a reflected wave. ${ }^{11}$ The drive energy scattered from the targets by these two instabilities back into the $f / 6.7$ optics of one cone 2 and cone 3 beam has been measured with calorimeters, and resolved spectrally and temporally with a full-aperture backscatter system (FABS). In total, as much as $5 \%$ (which is an upper bound) of the energy onto each face of the target is scattered, principally through the SBS channel. The calorimeter-measured reflected energy, as a fraction of total energy in a particular beam, is listed for each shot in Table 1. Analysis of the streaked spectra of the scattered energy is underway to diagnose plasma temperatures and densities. 

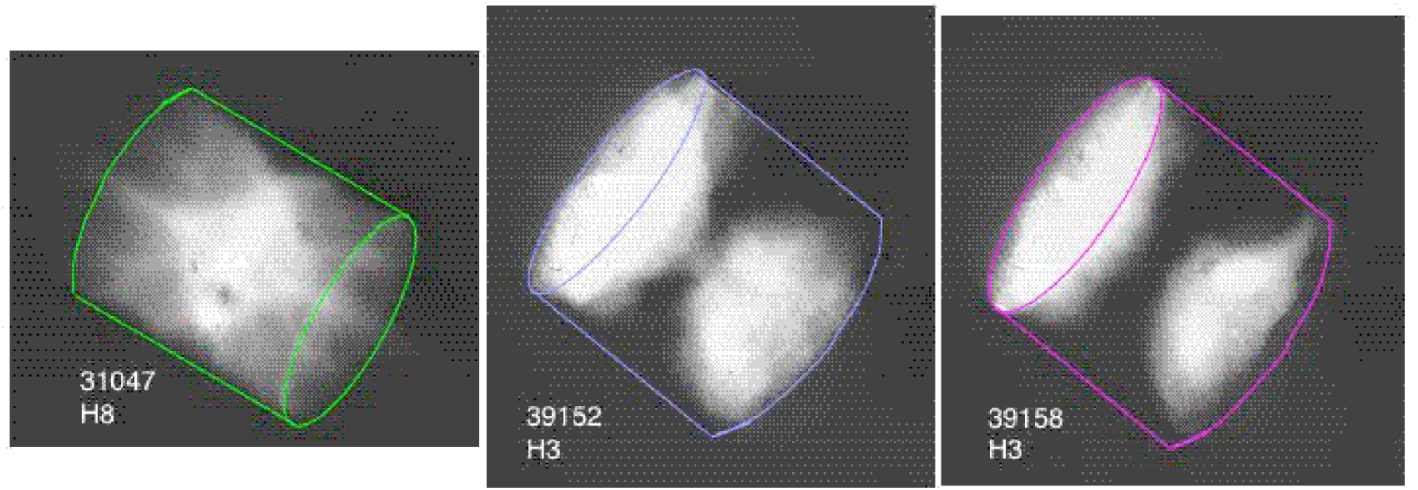

Figure 2. Time integrated x-ray pinhole images from three doped-aerogel targets with different densities. From left to right, the targets had densities of $3.1,4.8$ and $6.5 \mathrm{mg} / \mathrm{cc}$, which correspond to densities of $0.09,0.14$ and $0.19 n_{c r}$. The differences in axial extent of the x-ray emitting region is due to the difference in target density, and possibly also to differences in irradiation intensity and target chemistry.

\subsection{The X-Ray Source}

The x-ray source for this test series was a germanium-doped $\mathrm{SiO}_{2}$ aerogel that contained $50 \%$ Ge by weight $(20$ atomic percent). The aerogels as fielded were made at two densities, $4.8 \mathrm{mg} / \mathrm{cm}^{3}$ and $6.5 \mathrm{mg} / \mathrm{cm}^{3}$; these densities resulted in plasmas with electron densities 14 and 19\%, respectively, of the laser's critical density (critical density, $n_{c r}$, for $351 \mathrm{~nm}$ light is $\approx 9 \times 10^{21} \mathrm{~cm}^{-3}$ ). The $14 \%$ critical-density value for these targets was chosen based on experience with x-ray output from gas targets. ${ }^{7}$ The higher level of dopant possible in these targets compared to previous Ti-doped aerogels ${ }^{6}$ is due to the chemical similarity of Ge and Si. Unlike the case of the Ti dopant, where the $\mathrm{Ti}$ atoms are suspended and supported in the $\mathrm{SiO}_{2}$ matrix, the Ge atoms of the present targets are incorporated directly into the aerogel polymer chains. The aerogel material was cast in $75-\mu \mathrm{m}$ thick Be cylinders that were $1.2-\mathrm{mm}$ long and had inner diameters of $1.5 \mathrm{~mm}$. The Be walls of the cylinders transmit greater than $95 \%$ of the $10-\mathrm{keV}$ Ge x rays. The two open faces of the cylinders were each irradiated with up to $10 \mathrm{~kJ}$ of blue $(351 \mathrm{~nm})$ laser light. The beams penetrate the aerogel, supersonically ${ }^{3,12}$ or transonically, and heat to several kilo-electron volts ( $\mathrm{keV})$ a large fraction of the volume in the cylinder producing K-shell Ge ions $\left(\mathrm{Ge}^{30+}\right)$. The supersonic heating of the aerogel means that the laser energy goes to ionizing, locally, the high-Z x-ray emitting atoms, and not to ablating material; energy is not lost to large-scale hydrodynamic motion of the target. Thus, the laser energy is efficiently converted into $10-\mathrm{keV}$ Ge K-shell x rays. Time-integrated pinhole x-ray pictures of three of our targets are shown in Fig. 2. The pinhole cameras used for Fig. 2 were filtered with 11-mil Be, thereby restricting the images to $\mathrm{x}$ rays with energies greater than $\approx 3 \mathrm{keV}$. The image on the left is from a 3.1 $\mathrm{mg} / \mathrm{cm}^{3}$ Ti-doped aerogel in a $2.2 \times 2.0 \mathrm{~mm}$ (length $\times$ diameter) cylinder; thus it shows a heated volume that is $\sim 3.3 \times$ larger than those of the cylinders holding the Ge-doped aerogels used here. It is an open and interesting question as to why the emission seems to be constrained to two hot lobes of plasma near the faces of the cylinder for the two higher-density targets; these images suggest that the targets can be further optimized by using a lower aerogel density between $3-4.8 \mathrm{mg} / \mathrm{cm}^{3}$. Another unresolved question is whether conductive losses to the cylinder walls ${ }^{13}$ affect the propagation of the heat front, resulting in the forward-peaked axial profile seen in the middle image. These questions are being addressed through modeling and simulation.

The time-integrated x-ray spectrum of the heated target was measured between $1.5-15 \mathrm{keV}$ for each shot with a four-channel crystal spectrometer (HENWAY). ${ }^{14}$ The spectrometer had a view of one face of the target at an angle of $61^{\circ}$ with respect to the target axis. Two channels gave coverage in the stated energy band. The higher-energy channel used a pentaerythritol (PET) crystal, while the lower-energy channel was measured with a potassium-hydrogen-phthalate (KAP) crystal. The PET-crystal measured the spectrum between $4.5-15 \mathrm{keV}$. This channel was filtered differently across the face of the crystal: 1 or 3-mils Be, plus either 6 or $12-\mu \mathrm{m} \mathrm{Al}$, and strips of $25-\mu \mathrm{m} \mathrm{V}, 25.4-\mu \mathrm{m} \mathrm{Cu}$ or 1.8-mil Ge foils. The spectra were recorded on Kodak DEF film. Absorption edges in regions of the film filtered with the foils serve as energy fiducials allowing calibration of the positions of 


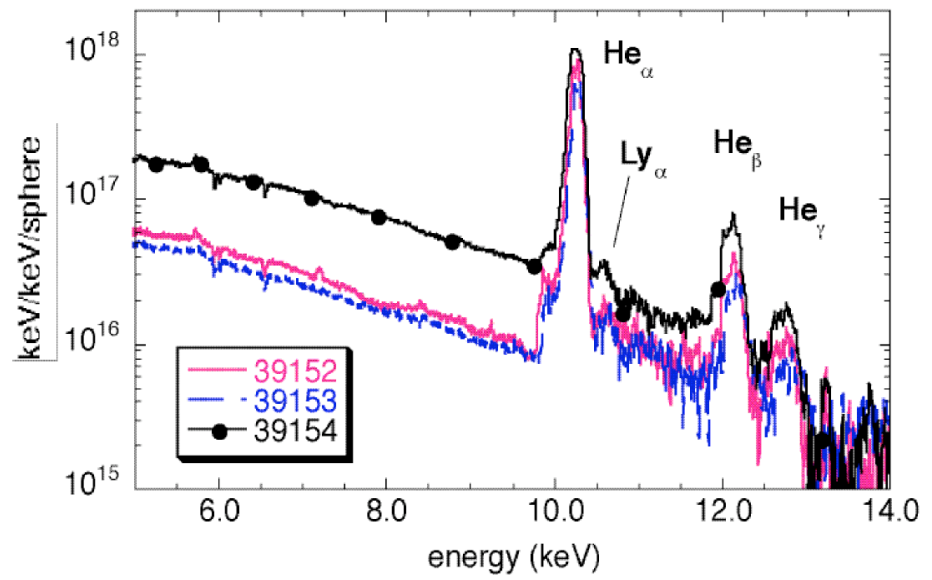

Figure 3. Three spectra from this test series (shots 39152, 39153 and 39154) measured with the PET crystal HENWAY channel (channel A). The targets for shots 39152 and 39154 were both 20 atom-percent Ge with density 4.8 mg/cc, shot $39153 \mathrm{had} 20$ atom-percent Ge and density $6.5 \mathrm{mg} / \mathrm{cc}$. The total energy on target for 39154 was $6 \%$ greater after resolving issues in three of the 40 drive beams.

measured lines. The spectra from three of the shots are shown in Fig. 3. The spectra shown are dominated by the $\mathrm{He}_{\alpha}(1 \mathrm{~s}-2 \mathrm{p})$ line of $\mathrm{Ge}^{30+}$ at $10.3 \mathrm{keV}$, but $\mathrm{He}_{\beta}(1 \mathrm{~s}-3 \mathrm{p})$ and $\mathrm{He}_{\gamma}(1 \mathrm{~s}-4 \mathrm{p})$ features are also visible above $12 \mathrm{keV}$. For five of the six shots in the present series, the continuum emission on the soft side of each spectrum accounts for less than $50 \%$ of the total emission in the $4.5-15 \mathrm{keV}$ spectral band, while for shot 39154 , the continuum is nearly $60 \%$ of the total output in the $4.5-15 \mathrm{keV}$ band (see Fig. 3). The continuum emission in each spectrum can be fit to an exponential to get a time-averaged approximate plasma-electron temperature; results for the the six shots of the present series ranged from $2-3 \mathrm{keV}$. The relative intensities of the $\mathrm{Ge}^{31+} \mathrm{Ly}_{\alpha}$ to $\mathrm{Ge}^{30+} \mathrm{He}_{\alpha}$ line features in Fig. 3 suggest temperatures in the target plasmas of $2.5-3.5 \mathrm{keV}$.

\subsection{Diagnostics}

Both time-integrated and temporally resolved $\mathrm{x}$-ray output measurements were made in this shot series; time integrated x-ray outputs were measured with the HENWAY spectrometer described above. An array of nine diamond photoconductive devices (PCDs) was provided by Sandia National Laboratory in order to measure the time history and yield of the x-ray pulses. ${ }^{15}$ The array was designed to fit in the boat of the OMEGA TIMs, and was fielded in TIM1. Several of the PCDs have also been used in radiation-effects tests on the SNL Z facility, and their responses are well known. The PCDs were filtered with 0.3 to 10 mils of kapton in order to discriminate contributions to the output from different regions of the radiated spectrum. The three PCDs filtered with 10 mils of kapton also had an additional $15.6 \mu \mathrm{m}$ of aluminum. Those channels measured x-rays above $4 \mathrm{keV}$, which is complementary with the spectral range measured by the high-energy channel of the HENWAY spectrometer. The PCD signals were recorded with the TDS6804 scopes (50-ps sampling), and redundant traces were read on TDS694C scopes provided by Sandia. The signals were sent to the scopes down $60 \mathrm{ft}$ of $3 / 8$ " heliax cable; bandwidth loss in the cables, which was significant, was quantified before the shots. All diagnostic information from the PCDs is frequency compensated according to the known bandwidth losses, and corrected for the PCD dc-bias response at large voltage responses.

A streaked crystal spectrometer was fielded to obtain time-resolved temperature measurements of the $\mathrm{x}$ ray source from diagnostic line ratios. This spectrometer was fielded in TIM2 with an ammonium dihydrogen phosphate (ADP) crystal and filtering of 15 mils of Be plus $12 \mu \mathrm{m} \mathrm{Al}$, but due to insufficient resolving power of the crystal, and a problem of persistence of the signal on the CsI photo-cathode, this diagnostic did not return useful data. As noted above, however, an estimate of $2-3 \mathrm{keV}$ for the average temperature of the x-ray emitting 

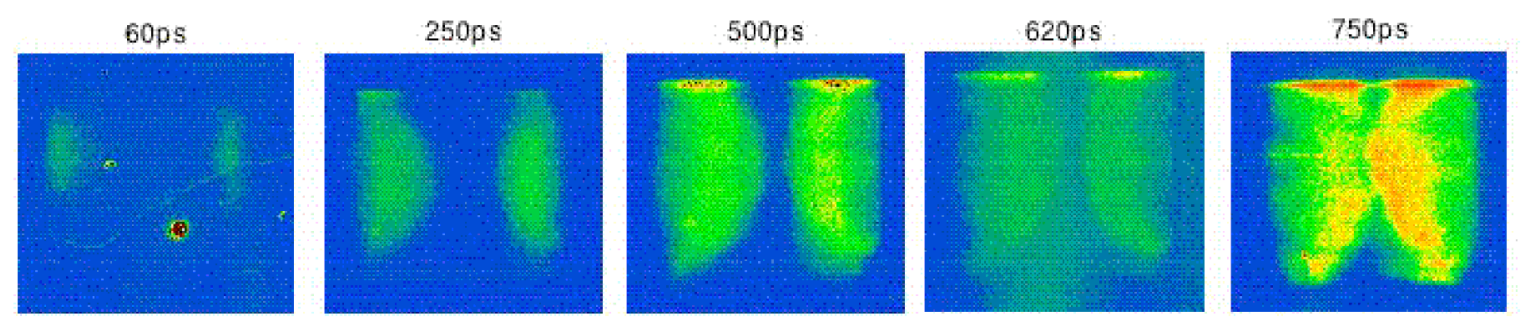

Figure 4. Five x-ray images from shot 39154 taken with XRFC4. The radial extent of the x-ray emitting region expands to the full diameter of the cylinder in under $500 \mathrm{ps}$, the heating fronts from the two faces of the cylinder meet at the targets center sometime near 750 ps after the start of the laser pulse.

plasma can be made from looking at the continuum signal on the high-energy channel from the HENWAY spectrometer.

The Ge-doped aerogel used to produce $x$ rays in the present shot series should be underdense to the $3 \omega$ drive laser. This means that the laser should be able to heat the entire volume of the source supersonically. In order to measure the extent of the laser heating, an x-ray framing camera (XRFC4) was fielded in TIM3. The camera was filtered with 15 mil of Be, which turned out to be slightly thinner than optimal. The camera has an array of $1650-\mu \mathrm{m}$ pinholes in front, which are imaged onto four strips of a microchannel plate (MCP) detector. The camera and detector were rotated $30^{\circ}$ in the TIM in order to have the axis of the MCP strips parallel to the axis of the cylinder holding the doped aerogel. The camera had a $4 \times$ magnification, which fit the $1.5 \mathrm{~mm}$ diameter aerogel target onto the $7 \mathrm{~mm}$ width of the MCP strips of the XRFC4. Five images from shot 39154 are shown in Fig. 4. The images show that the aerogel heats quickly in the radial direction, which is closer to the direction of propagation of the steeper-angle beam cones, but takes approximately 750 ps for the heat fronts from the two faces of the cylinder to meet in the axial direction. This is slower than was previously observed ${ }^{12}$ with lower-density aerogels, but is consistent with the understanding of underdense heating phenomena. Shot 39154 was the only one where the two heat fronts overlapped during the laser pulse. From Fig. 3 (and Table II), this is also the shot with the greatest output. This observation indicates that optimal target performance can be achieved by either reducing the aerogel density, or shrinking the target, both of which should enable the heating fronts to overlap more easily. Work in these directions will be pursued in future source-development experiments.

Thermoelastic calorimeters (TECs) were also used to measure the x-ray yield of the target; they were fielded on four shots in TIM5. The TECs use the piezoelectric properties of quartz to convert a stress wave into a voltage signal. A disk of 6061-T6 aluminum was attached with epoxy to a quartz disk that had electrodes attached. The absorbing surface of the $\mathrm{Al}$ was positioned $9 \mathrm{~cm}$ from the $\mathrm{x}$-ray-emitting aerogel plasma. The x-ray deposition in the $\mathrm{Al}$ produces a stress wave in the $\mathrm{Al}$ disk that propagates in the quartz. The stress in the quartz produces a voltage response proportional to the stress. Two different filters ( 2 mil kapton and 10 mil kapton) were used to examine the response for different spectra and different fluences. At the fluences produced, the filters at their $8 \mathrm{~cm}$ position ( $1 \mathrm{~cm}$ in front of the Al plate) were ablated and deformed to the point where maintaining their position was problematic. They also were not reusable.

\section{DATA ANALYSIS}

\subsection{The X-Ray Source}

The measured total yields (KAP and PET channels summed) and the yields in the 4.5-15 keV range from the PET HENWAY channel for all six of our x-ray-source targets are given in Table 2. The majority of the energy that is radiated is in the soft spectral range monitored by the KAP crystal. Uncertainty in the crystal reflectivities, the filter transmission and the detector response (Kodak DEF film) combine for a $30 \%$ uncertainty 
Table 2. Yields from Ge-doped aerogels: columns are shot number, target density, total yield from KAP and PET HENWAY channels (summed), yield between 4.5-15 keV from HENWAY PET channel, yield with $h \nu>4 \mathrm{keV}$ from the PCDs, yield with $h \nu \gtrsim 4 \mathrm{keV}$ for shots 39156, 39157 from the TECs, or (underlined) total x rays from the source estimated by the TEC response $(39154,398158)$, the average yield ' $\mathrm{Y}$ ' in the $h \nu \gtrsim 4.5 \mathrm{keV}$ band (HENWAY PET and PCD) and resulting conversion efficiencies (CE).

\begin{tabular}{lcrrrrrr}
\hline \hline shot no. & $\rho(\mathrm{mg} / \mathrm{cc})$ & HENWAY total $(\mathrm{J})$ & PET $(\mathrm{J})$ & PCD $(\mathrm{J})$ & TEC $(\mathrm{J})$ & Y $(\mathrm{J})$ & $\mathrm{CE}(\%)$ \\
\hline 39152 & 4.8 & 2207 & 105.6 & & & 105.6 & 0.61 \\
39154 & 4.8 & 6460 & 269.9 & 247.8 & $\underline{11260}$ & 258.9 & 1.41 \\
39156 & 4.8 & 2374 & 140.2 & 152.1 & 61.8 & 146.2 & 0.80 \\
39157 & 4.8 & 3097 & 145.2 & 159.9 & 88.3 & 152.6 & 0.81 \\
39153 & 6.5 & 1652 & 85.6 & 79.8 & & 82.7 & 0.47 \\
39158 & 6.5 & 1956 & 98.1 & 113.1 & $\underline{2690}$ & 105.6 & 0.57 \\
\hline \hline
\end{tabular}

in the stated HENWAY yields. The x-ray yields estimated from the most heavily filtered PCD channels (10 mils kapton plus $15.6 \mu \mathrm{m} \mathrm{Al}$ ) and the thermoelastic calorimeters are given in the fifth and sixth columns of Table 2, respectively. The $\mathrm{PCD}$-measured yields are the average of three signals; statistical variation and uncertainty in the PCD response combine for a $25 \%$ uncertainty. The PCD yields show trends that follow the trends in the HENWAY yields closely, and are in excellent agreement with the absolute values of the (PET-measured) output for each shot. The TEC numbers show the same trends as the yields measured with HENWAY, however, the X-ray yields for the two shots where the TECs were filtered with 10 mil of kapton (39156 and 39157) are $\approx 40-60 \%$ below both the PCD and HENWAY (PET) yields. From the data in Table 2 one can note that the two higher density targets (shots 39153 and $39158, \rho=6.5 \mathrm{mg} / \mathrm{cc}$ ) had the lowest yields, confirming that the optimal target density is closer to $\rho=4.5 \mathrm{mg} / \mathrm{cc}$ in this case. ${ }^{7}$ A lower-density, Ge-doped target has not been shot, so we cannot conclude that $\rho=4.8 \mathrm{mg} / \mathrm{cc}$ is the optimum density for these sources.

Analysis of the softer-energy HENWAY channel (KAP crystal, 1.5-3.5 keV) is in progress, but derived yields are in the ranges of $1.5-1.8$ to $2.5-6.1 \mathrm{~kJ}$ for the 6.5 and $4.8 \mathrm{mg} / \mathrm{cc}$ targets, respectively (third column in Table 2). The spectra from this channel will give information about the total power budget for conversion of the laser energy into x rays, and possibly plasma-temperature information if diagnostic line ratios can be found. In the cases of the two lightly filtered TEC gauges (39154 and 39158), the sum of the softer- and harder-energy HENWAY channels is $30-40 \%$ below the total yield derived from the TEC response. However, in the case of shot 39154 , the TEC gauge estimates that $50 \%$ of the total laser energy comes out in the x-ray yield, which is unlikely. Note, with appropriate filtering, the softer-x-ray output from these targets can be removed from the radiation used for diagnostic applications.

The measured x-ray waveform as given by the PCDs is compared to the laser power delivered to the face of the target for shot 39156 in Fig. 5. The time of the peak of the x-ray pulse has been arbitrarily matched to the time of the peak of the laser. The leading edge of the x-ray pulse lags the leading edge of the laser pulse slightly, which is due to the time it takes for the laser to ionize and heat the aerogel material; the x-ray pulse extends slightly past the laser pulse since the hot plasma still emits $x$ rays as it cools. The x-ray waveform shown has an $\approx 700 \mathrm{ps}$ rising edge and a $1.0 \mathrm{~ns}$ fwhm. Note that the filter assembly used for these shots was fielded on all shots with no changes, and no evidence of damage was observed, reinforcing that the laser driver produced very little debris. Given the representative spectra in Fig. 3, and the yields in Table 2, we can compute the fluence seen by a test object. Taking only the data for the $4.8 \mathrm{mg} / \mathrm{cc}$ aerogels, and looking at shots 39152 and 39154 as the lower and upper limits on output, then fluences were between 0.025 and $0.061 \mathrm{cal} / \mathrm{cm}^{2}$ for the $9 \mathrm{~cm}$ stand-off distance of the TEC gauges. The mean-photon energy in the spectra providing the $\mathrm{x}$-ray fluence is $\approx 7 \mathrm{keV}$.

\section{SIMULATIONS}

We have run two-dimensional, Lagrangian, radiation-hydrodynamical pre-shot simulations of our laser-heated aerogel targets with the LASNEX code. ${ }^{16,17}$ The simulations have three cones of beams onto both faces of the 


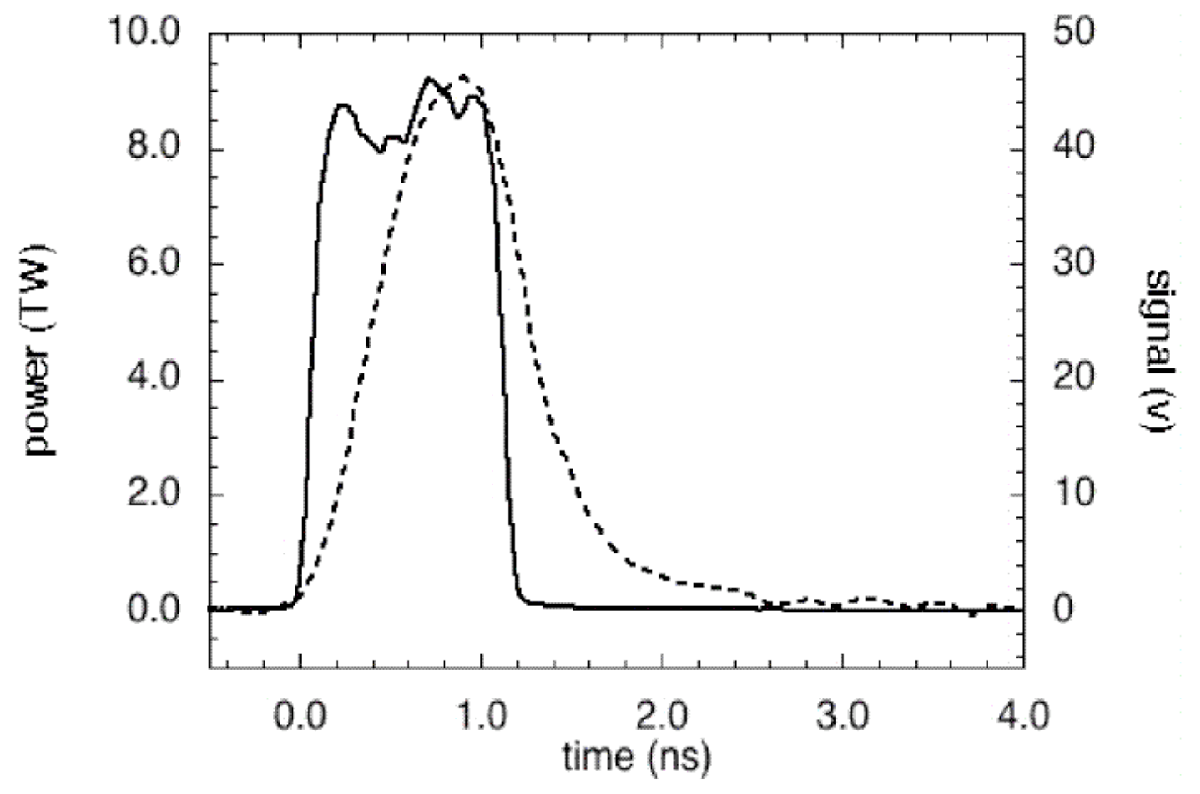

Figure 5. Laser power (solid trace, left scale) delivered to the face of the cylindrical target seen by the PCD array for shot 39156, and the x-ray waveform (dashed trace, right scale) measured by the PCDs (number 50, filtered with 10 mil kapton and $15.6 \mu \mathrm{m} \mathrm{Al})$.

aerogel cylinder at angles of 21.4, 42.0 and 58.9 degrees with respect to the normal to the face. The focal spot is a super-Gaussian with 2000 rays distributed across the spot's face. Ray-tracing techniques are used to model the laser propagation in the target, and inverse bremsstrahlung is the means by which laser energy is deposited. The simulations were run with an average energy of $490 \mathrm{~J}$ per beam, which delivers $19.6 \mathrm{~kJ}$ to the target, slightly higher than what was actually shot. In the simulation, the laser energy was delivered in a 1 ns square pulse with a $150 \mathrm{ps}$ linear-ramp rise and fall. The resulting peak intensities in the simulation was $\approx 2 \times 10^{16} \mathrm{~W} / \mathrm{cm}^{-2}$ on the target's axis. Electron heat transport in the targets is described by a standard multigroup flux-limited heat-diffusion model, in which the heat flux in a give zone is the minimum quantity of either the Spitzer-Härm thermal conductivity ${ }^{18}$ or the flux-limited product of the electron thermal sound speed, electron density and temperature. A finite-element treatment of both the electron heat conduction and radiation diffusion has been employed, this gives a more accurate solution to the conduction equations for distorted Lagrangian meshes. We have previously investigated the supersonic heat-front propagation in underdense aerogel targets ${ }^{12}$ and have found that for intensities an order of magnitude lower than in the present work, the standard heat-conduction model in LASNEX is adequate for predicting the heating in these targets as a function of time. However, increasing the laser intensity by a factor of two (in targets that were $35 \%$ less dense than the present targets) required a model that accounted for non-local electron heat transport in order to match measured heat-front trajectories. The present experiments have been carried out in an intensity regime that is $4 \times$ higher than this. Given this caveat, the simulations predict a merging of the heated volumes formed at each face of the $1.2 \mathrm{~mm}$ long target by sometime between 200 and $300 \mathrm{ps}$ after the start of the laser pulse. From the framing camera images taken on each shot, merging of the heated regions is almost never seen during the laser pulse. For only one shot (39154, Fig. 4) do the heated regions even merge, and then at $\approx 750$ ps after the start of the pulse.

The Detailed Configuration Accounting (DCA) atomic physics package ${ }^{19}$ was used in LASNEX to compute the radiative emission from silicon, oxygen and germanium. The DCA package solves rate equations for the number of ions in each important excited state in each ionization state. This package is used when accurate atomic physics is needed for line diagnostics. DCA can handle any number of states connected by radiative and collisional bound-bound and bound-free, and auto-ionization and dielectronic recombination processes. The states and transition rates are specified in data files generated by other codes. Optionally, a simple screened hydrogenic 
Table 3. LASNEX spectral-output prediction results.

\begin{tabular}{lc}
\hline \hline Energy band (keV) & Yield $(\mathrm{kJ})$ \\
\hline $0-1.15$ & 2.25 \\
$1.15-3.99$ & 2.42 \\
$3.99-9.37$ & 0.36 \\
$9.37-16$ & 0.16 \\
$0-16$ & 5.19 \\
\hline \hline
\end{tabular}

model can be produced at problem initialization. This latter option was used in the present simulations.

Peak temperatures in the simulation of the heated cylinder were $\gtrsim 4 \mathrm{keV}$, which is consistent with the appearance of the $\mathrm{Ge}^{31+} \mathrm{Ly}_{\alpha}$ feature in some of the observed spectra (see Fig. 3). The predicted spectral yield from the target is given in Table 3 . The predicted yield in the $1.15-4 \mathrm{keV}$ spectral band, which is dominated by free-free continuum, Si K-shell lines and L-shell transitions in L- and K-shell Ge ions, is reasonably close to what was measured with the HENWAY KAP channel for three of our low-density shots, but far below the target output from the highest-performing shot (39154). The predicted yield between $4-15 \mathrm{keV}$ is $\approx 520 \mathrm{~J}$ into $4 \pi$ steradian; this is $\approx 2 \times$ greater than the yield in the same band for the highest performing shot of this campaign. It is not clear if a more sophisticated atomic model, or a better treatment of the electron heat-conduction in the problem would bring the predicted yield from the target more in line with the measurements.

\section{CONCLUSIONS}

Large x-ray outputs from Ge-doped aerogels have been measured in the $4.5-15 \mathrm{keV}$ band. The observed conversion efficiencies average $0.9 \%$ for the four $\rho=4.8 \mathrm{mg} / \mathrm{cm}^{3}$ targets of the present shot series; this is higher than what has traditionally been measured from solid-foil targets in this energy band. Minimal energy is reflected from the target $(\lesssim 5 \%)$ indicating that most of the laser energy is available to couple to our underdense aerogels. Framing camera images show a quick heating in the radial direction of our cylindrical targets, but in only one of our targets did the heat fronts from the two faces of the target merge at the center of the target; that one target strongly outperformed the other targets with similar densities. Our x-ray yields for the targets are consistent on three independent diagnostics, a crystal spectrometer, PCDs and thermoelastic calorimeters; the PCD data indicate that the history of the X-ray output tracks the delivered laser power. Simulations made with the LASNEX code over predict x-ray output in the $4-15 \mathrm{keV}$ band; it is known that in the laser-intensity regime in which these experiments take place, non-local heat-transport effects are important (if not dominant) and must be taken into account. Finally, it is clear that optimization remains to be done on the targets that have been fielded: a true optimum density remains to be found, targets less dense than $4.8 \mathrm{mg} / \mathrm{cm}^{3}$ should be shot; perhaps better laser coupling will be found at lower laser intensities (larger spot sizes on the target); and shrinking the axial dimension of the target may result in better performance by ensuring the heat fronts can overlap.

\section{ACKNOWLEDGMENTS}

The authors would like to thank the entire crew at the OMEGA laser for their expert operation of the laser and help setting up these experiments. In particular, Dr. Vladimir Glebov and Raymond Bahr were particularly helpful. We would also like to thank Ms. Norine Zuchowski at Sandia National Laboratories for her help fabricating the cable plant, and James Tellinghusen, Chuck Source and Vern Rekow at Lawrence Livermore National Lab for their help in machining parts and setting up for this experiment. This work was performed under the auspices of the U. S. Department of Energy by Lawrence Livermore National Laboratory under Contract No. W-7405ENG-48. Sandia is a multi-program laboratory operated by Sandia Corporation, a Lockheed Martin Company, for the U.S. Department of Energy under Contract DE-AC04-94AL8500. 


\section{REFERENCES}

1. O. L. Landen, D. R. Farley, S. G. Glendinning, L. M. Logory, P. M. Bell, J. A. Koch, F. D. Lee, D. K. Bradley, D. H. Kalantar, C. A. Back, and R. E. Turner, "X-ray backlighting for the national ignition facility (invited)," Rev. Sci. Instrum. 72, pp. 627-633, 2001.

2. J. Workman and G. A. Kyrala, "X-ray yield scaling studies performed on the OMEGA laser," Rev. Sci. Instru. 72, pp. 678-681, 2001.

3. J. Denavit and D. W. Phillion, "Laser ionizatoin and heating of gas targets for long-scale-length instability experiments," Phys. Plasmas 1, pp. 1971-1984, 1994.

4. C. A. Back, J. Grun, C. Decker, L. J. Suter, J. Davis, O. L. Landen, R. Wallace, W. W. Hsing, J. M. Laming, U. Feldman, M. C. Miller, and C. Wuest, "Efficient multi-kev underdense laser-produced plasma radiators," Phys. Rev. Lett. 87, p. 275003, 2001.

5. C. A. Back, J. Davis, J. Grun, L. J. Suter, O. L. Landen, W. W. Hsing, and M. C. Miller, "Multi-kev x-ray conversion efficiency in laser-produced plasmas," Phys. Plasmas 10, pp. 2047-2055, 2003.

6. K. B. Fournier, C. Constantin, J. Poco, M. C. Miller, C. A. Back, L. J. Suter, J. Satcher, J. Davis, and J. Grun, "Efficient multi-kev x-ray sources from ti-doped aerogel targets," Phys. Rev. Lett. 92, p. 165005, 2004.

7. K. B. Fournier, C. Constantin, C. A. Back, L. Suter, H.-K. Chung, M. C. Miller, D. H. Froula, G. Gregori, S. H. Glenzer, and O. L. Landen, "Electron-density scaling of conversion efficiency of laser energy into l-shell x-rays," J. Quant. Spect. and Rad. Transf. accepted, to appear, 2005.

8. F. Girard et al., "Multi-kev x-ray conversion from pre-pulsed foil experiments," in Laser-Generated 84 Other Lab. X-ray 8 EUV Sources, Optics, Apps., G. A. Kyrala, J.-C. J. Gauthier, C. A. MacDonald, and A. M. Khounsary, eds., Proc. SPIE 5196, pp. 220-232, 2004.

9. F. Girard et al., "Experimental multi-kev x-ray conversion efficiencies from laser exploded germanium foil (P1.155)," in 32 $2^{\text {nd }}$ EPS Conference on Plasma Physics, (Tarragona, Spain), 27 June - 1 July 2005.

10. T. R. Boehly et al., "The upgrade to the OMEGA laser system," Rev. Sci. Instru. 66, pp. 508-510, 1995.

11. W. L. Kruer, The Physics of Laser Plasma Interactions, Westview Press, Boulder, 2003.

12. C. Constantin, C. A. Back, K. B. Fournier, M. C. Miller, E. L. Dewald, and O. Landen, "Experimental investigations of supersonic heat-wave propagation in laser-produced underdense plasmas," Phys. Plasmas 12, p. 063104, 2005.

13. C. A. Back, J. D. Bauer, J. H. Hammer, B. F. Lasinski, R. E. Turner, P. W. Rambo, O. L. Landen, L. J. Suter, M. D. Rosen, and W. W. Hsing, "Diffusive, supersonic x-ray transport in radiatively heated foam cylinders," Phys. Plasmas 7, pp. 2126-2134, 2000.

14. L. N. Koppel and J. D. Eckels, "High resolution x-ray crystal spectrographs," Lawrence Livermore National Laboratory Report, UCRL-79781, October, 1977.

15. R. B. Spielman, "Diamond photoconducting detectors as high-power z-pinch diagnostics," Rev. Sci. Instr. 66, pp. 867-870, 1995.

16. G. Zimmerman and W. Kruer, "Numerical simulation of laser-initiated fusion," Comments Plasma Phys. Control. Fusion 2, pp. 51-61, 1975.

17. J. A. Harte, W. E. Alley, D. S. Bailey, J. L. Eddleman, and G. B. Zimmerman, "LASNEX - a 2-d physics code for modeling ICF," in ICF Quarterly Report, M. Marinak, ed., pp. 150-164, Lawrence Livermore National Laboratory, UCRL-LR-105821-96-4, July-September 1996.

18. L. Spitzer and R. Härm, "Transport phenomena in a completely ionized gas," Phys. Rev. 89, pp. 977-981, 1953.

19. Y. T. Lee, "A model for ionization balance and L-shell spectroscopy of non-LTE plasmas," J. Quant. Spectrosc. Radiat. Transf. 38, pp. 131-145, 1987. 